\title{
Features of the mammal marl transposons in the human, sheep, cow, and mouse genomes and implications for their evolution
}

\author{
Marie-Véronique Demattei, ${ }^{1}$ Corrine Augé-Gouillou, ${ }^{1}$ Nicolas Pollet, ${ }^{2}$ Marie-Hélène Hamelin, ${ }^{1}$ \\ Michèle Meunier-Rotival, ${ }^{2}{ }^{1}$ ves Bigot ${ }^{1}$ \\ ${ }^{1}$ I.R.B.I., Groupe d'Etude des Parasites Moléculaires, UPRESA CNRS 6035, Faculté des Sciences, Parc Grandmont, 37200 Tours, France \\ ${ }^{2}$ INSERM U347 affiliée au CNRS, 80 Rue du Général Leclerc, 94276 Le Kremlin Bicêtre Cedex, France
}

Received: 30 March 2000 / Accepted: 25 July 2000

Abstract. Mariner-like elements (MLE) belong to the Tc1/ mariner superfamily of class II transposons. We have analyzed the mariner related to the cecropia subfamily, and called mammal marl, in four mammalian genomes, Bos taurus (Bovidae), Homo sapiens (Primata), Mus musculus (Rodentia), and Ovis aries (Ovidae). Three kinds of MLE sequences were found in all these species: full-length 1.3-kbp elements, shorter elements $80 \mathrm{bp}-1.2 \mathrm{kbp}$, and single inverted terminal repeats (ITRs). All the 1.3-kbp genomic copies sequenced had an open reading frame encoding a transposase interrupted by stop codons or frame shifts. Phylogenetic analysis of the full-length elements suggested at least two distinct populations of mammal marl elements in each species. This was confirmed by using a statistical method that allows defining populations. Finally, the evolutionary origin of the mammal marl elements and the paradoxes are discussed.

\section{Introduction}

The mariner-like elements (MLEs) are members of a diverse and taxonomically widespread superfamily of eukaryotic transposons that are present in both vertebrates and invertebrates (for review, see Plasterk et al. 1999). They are small elements about 1280 bp long and are flanked by inverted terminal repeats (ITRs), each 28-30 bp long. These elements move about in genomes by a conservative cut-and-paste transposition, similar to that described for several bacterial insertion sequences (reviewed by Mahillon and Chandler 1998). This process occurs via a DNA intermediate and is catalyzed by a single transposase encoded by the transposon. Whereas MLEs are widespread in the eukaryotes, very few contain an effective open reading frame encoding the transposase, because of the presence of stop codons and frameshifts. All the functional natural elements found so far are related to the Mos-1 element isolated from Drosophila mauritiana (Maruyama et al. 1991).

MLEs were found in mammalian genomes in 1995 when two MLE "species" related to the cecropia and irritans subfamilies were discovered in the human genome, and named Hsmarl and Hsmar2, respectively (Augé-Gouillou et al. 1995; Oosumi et al. 1995).

The Hsmarl consensus element (Acc\# U52077) is $1287 \mathrm{bp}$ long, with 30-bp perfect ITRs, and encodes a 343 amino acid transposase (Morgan 1995). About 200 copies of Hsmarl, 2400 copies of 80-bp paired ITR structures, and 4600 copies of single ITRs are believed to be present in the human genome based on the databank analyses. There are mariner elements closely related to Hsmarl elements in the genomes of primates, sheep, and cows, but they seem to be absent from Scandentia, Dermoptera and Rodentia. This suggested that these MLEs entered mammalian genomes a very long time ago, or on several occasions. Robertson and

Correspondence to: Y. Bigot; E-mail: bigot@univ-tours.fr
Zumpano (1997) propose that the ancestor of the Hsmarl in the human genome gave rise to a single lineage of Hsmarl elements and was formed about 50 million years (Myr) ago.

The present study focuses on the Hsmarl subfamily in four mammalian genomes: Homo sapiens (human), Mus musculus (mouse), Ovis aries (sheep) and Bos taurus (cattle). They are called Hsmarl (human), Mmmarl (mouse), Oamarl (sheep) and Btmarl (cattle), and this lineage of elements is referred to as mammal marl. Their consensus sequences, diversity, numbers of MLEs and ITRs, and nucleotide divergence were all investigated by using sequences obtained by polymerase chain reaction (PCR) amplification or extracted from the databanks in order to improve our understanding of their evolution.

\section{Materials and methods}

Nucleic acid analysis. Genomic DNAs from Homo sapiens (white European), Bos taurus (Holstein race), and Ovis aries (Ile de France race) were obtained from sperm and lymphocyte samples of one male. Mus musculus DNA (Balb-c strain) was obtained from the lymphocytes of males. DNA purifications, digestions with restriction enzymes, agarose gel electrophoresis, and Southern blots were all performed as in AugéGouillou et al. (1995). The DNA probes were labeled with $\left[\alpha^{32} \mathrm{P}\right]$ dATP (ICN products) by primer extension using the Klenow fragment of DNA polymerase I and the oligonucleotides with $\left[{ }^{32} \mathrm{P}\right]-\gamma \mathrm{ATP}$ and $\mathrm{T} 4$ polynucleotide kinase (ICN products and Promega). Hybridizations were performed in $0.5 \mathrm{M} \mathrm{Na} 2 \mathrm{HPO}_{4}-\mathrm{NaH}_{2} \mathrm{PO}_{4}, 7 \%$ SDS, incubated overnight at $65^{\circ} \mathrm{C}$. The Southern blot membranes (NylonN+, ICN products) were washed with SSC $(1 \times \mathrm{SSC}=0.15 \mathrm{M} \mathrm{NaCl}, 0.015 \mathrm{~m}$ trisodium citrate $)$, at $65^{\circ} \mathrm{C}$.

Dot blot estimation of the number of copies of mammal mar1 elements and ITR in genomes. Dot blots of genomic DNA and a mix of full-length mammal marl clones linearized with EcoRI (Acc\# AJ009227-AJ009236 for human, Acc\# AJ009242-AJ009246 for sheep, Acc\# AJ009223-AJ009226 plus AJ009247 for cow, Acc\# AJ009237AJ009241 for mouse) were performed on NylonN+ (ICN products). Genomic DNAs from Escherichia coli and Drosophila melanogaster were used as negative hybridization controls. The concentration of DNA was determined assuming that $1 \mathrm{~A}_{260}=50 \mu \mathrm{g} / \mathrm{ml}$. Hsmarl, Oamarl, Btmarl, and Mmmarl clones were used as references for quantification. The amounts of Hsmarl elements were quantified by probing the membranes with a mix of five Hsmarl DNA fragments (Acc\# X84285-X84289) for the human DNA sperm. Similarly, the probes for membranes containing sheep or cow sperm DNA were a mix of six Oamarl or Btmarl DNA fragments (Acc\# X84290-X84295 or Acc\# 84296-X84301). The membranes containing DNA from mouse lymphocytes were probed with a mix of three probes. The amounts of mammal marl ITRs in the mammalian genomes were quantified with a single-stranded oligonucleotide $\left(5^{\prime}\right.$ TATTAGGTTGGTGCAAAAGT-3') as probe. Hybridizations were done in $0.5 \mathrm{M} \mathrm{Na}_{2} \mathrm{HPO}_{4}-\mathrm{NaH}_{2} \mathrm{PO}_{4}, 7 \%$ SDS, overnight at $65^{\circ} \mathrm{C}$ or at $42^{\circ} \mathrm{C}$. The membranes were washed with a final washing in $2 \times \mathrm{SSC}, 0.1 \%$ SDS at $65^{\circ} \mathrm{C}$ or in $6 \times \mathrm{SSC}, 0.1 \%$ SDS at $42^{\circ} \mathrm{C}$ with the oligonucleotide probe. Hybridized DNA was quantified with a Direct Imager (Packard). 
a.

b.

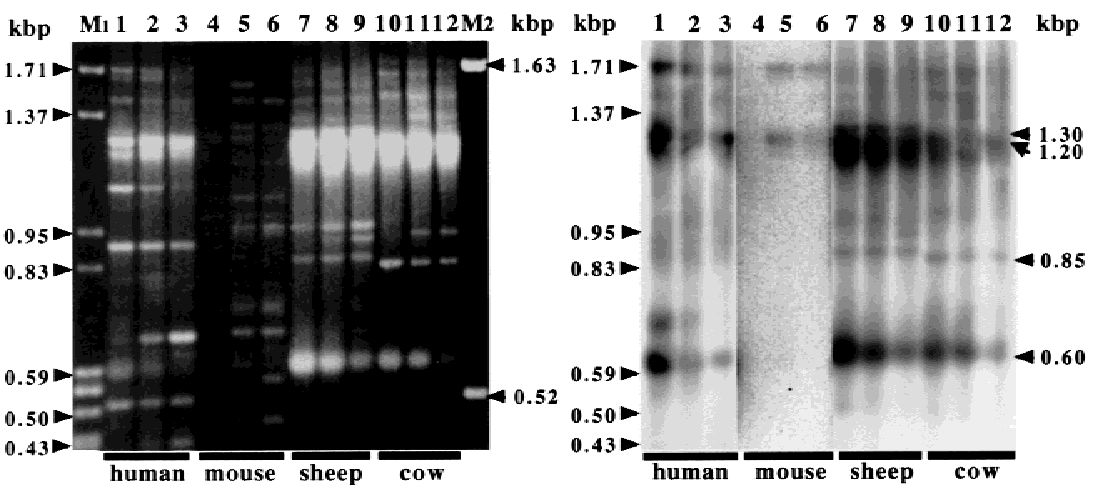

Fig. 1. Amplification of $H$. sapiens (lanes 1-3), M. musculus (lanes 4-6), O. aries (lanes 7-9), and $B$. taurus (lanes 10-12) genomic DNA by using a 20-mer primer corresponding to the ITR of Robertson's Hsmarl consensus sequence. Genomic DNA was from sperm (lane 1-3 and 7-12) and from blood (lane 4-6). No differences were observed when DNA from blood was used for $H$. sapiens, $O$. aries and $B$. taurus. (a) Amplification products were separated by electrophoresis (1.5\% agarose gel), stained with ethidium bromide, and photographed. (b) Southern blots of the agarose gel were probed with a mix of five fragments originating from Hsmarl clones (Acc\# X84285-X84289; lanes 1-3), a mix of six DNA fragments purified from Oamarl clones (Acc\# X84290-X84295; lanes 7-9) and a mix of six DNA fragments purified from Btmarl-related clones (Acc\# 84296-X84301; lanes 10-12). The 17 fragments were mixed to probe the mouse amplification products (lanes 4-6). $\mathrm{MgCl}_{2}$ concentrations: $1.5 \mathrm{~mm}$ (lanes 1, 4, 7, and 10), 2.5 mM (lanes 2, 5, 8, and 11), and $3.5 \mathrm{~mm}$ (lanes 3, 6,9 , and 12). The molecular weight markers are in lanes M1 and M2, and the sizes (in kbp) are indicated in the left- and right-hand margins.
PCR amplifications and fragment cloning. Polymerase chain reactions (PCR) were performed on $50 \mathrm{ng}$ genomic DNA. PCR reactions were done in $10 \mathrm{~mm}$ Tris- $\mathrm{HCl}, \mathrm{pH} 9 / 1.5 \mathrm{~mm} \mathrm{MgCl}_{2} / 50 \mathrm{~mm} \mathrm{KCl} / 0.1 \%$ TritonX100/150 mM each dNTP/0.1 mM of the oligonucleotide $\left(5^{\prime}\right.$ TATTAGGTTGGTGCAAAAGT- $3^{\prime}$ ) in a $100-\mu 1$ reaction volume with 2 units Taq DNA Polymerase (Promega). Each PCR was carried out in a programmable thermal controller (Perkin-Elmer) for 30 cycles. The cycle was: denaturation at $94^{\circ} \mathrm{C}$ for $1 \mathrm{~min}$, annealing at $53^{\circ} \mathrm{C}$ for $1 \mathrm{~min}$, and extension at $72^{\circ} \mathrm{C}$ for $1 \mathrm{~min}$. At the end of the $30^{\text {th }}$ cycle, the heat denaturation step was omitted, and extension was allowed to proceed at $72^{\circ} \mathrm{C}$ for 10 min. PCR fragments were eluted from agarose gels with a Gene Clean Kit (BIO 101) and inserted into the pGEM-T vector (Promega).

DNA sequencing reactions. Plasmids were purified and sequenced as previously described (Rabouam et al. 1999). The 25 sequences reported in this paper appear in the DDBJ/EMBL/GenBank sequence database under accession numbers AJ009223-AJ009247. The four consensus sequences reported in this paper, Cons $\mathrm{Hs}$, Cons $\mathrm{Bt}$, Cons $\mathrm{Oa}$, and Cons $\mathrm{Mm}$, are deposited in the DDBJ/EMBL/GenBank sequence database with the complete alignments under accession number DS34749.

Sequence analyses. The Infobiogen facilities (Dessen et al. 1990) were used for GenBank searches, extractions, sequence alignments and calculations. GenBank release 114 was used for searches and extractions of MLE sequences. The $\mathrm{CpG}$ dinucleotide frequencies were checked by calculating the ratio of observed $\mathrm{CpG}$ frequency:expected $\mathrm{CpG}$ frequency ( $\mathrm{CpG}$ obs/exp). For each investigated sequence, the expected $\mathrm{CpG}$ frequency was calculated from the $\mathrm{G}$ and $\mathrm{C}$ contents. Thus, the expected $\mathrm{CpG}$ frequency should be: $21 \times 15=3.1 \%$ for a DNA having a $\mathrm{G}$ content of $21 \%$ and a C content of $15 \%$.

Statistical treatment. The density ellipses reflecting the features of each MLE population in the mammalian genomes were defined, computed, and drawn from the distribution of $\mathrm{CpG}$ obs/exp related to the similarity percentage of each MLE sequence with their consensus. Their shape and area described the distribution in each defined MLE population with a probability equivalent to $95 \%$ (black line) and 99\% (grey line) confidence limit. Before computing the density ellipse, the bivariate normal distribution was tested for normality (Shapiro-Wilk W test at 0.05 significance; SAS Institute 1995).

\section{Results}

Size and number of copies of mammal mar1 elements. Previous studies on mammal marl elements have revealed a 10-20\% di- vergence between the sequences of mammal species (Robertson and Zumpato 1997). This sequence variation, which also alters restriction enzyme cleavage sites, prevents any study of the pattern of full-length and deleted elements in genomes by Southern blot/ hybridization experiments with genomic DNA digests. This was, therefore, done by PCR with the ITR sequence of the consensus sequence, AccN\# U52077, to design a primer.

Several PCR products, $0.43-1.3 \mathrm{kbp}$ long, were detected on agarose gels in the four species (Fig. 1a). However, most of them did not hybridize with the mammal marl probes. Their presence in the final PCR products was not owing to a setting deficiency of the optimal amplification conditions. They probably resulted from the presence nearby of single ITRs, which were used as annealing sites during PCR, thus allowing the amplification of these non-MLE fragments. Similar PCR results were obtained with DNA from sperm or lymphocytes from human, cow, and sheep, thus suggesting that there were no differences between these two tissues. This was confirmed with Southern blot/hybridization investigations by using restriction enzymes insensitive to methylated cytosine (data not shown).

Three main forms of mammal marl fragments were identified in the human, sheep, and cow samples: $1.3 \mathrm{kbp}, 1.2 \mathrm{kbp}$, and 0.6 $\mathrm{kbp}$ (Fig. 1b). Only the 1.3-kbp form was found in the mouse samples (Fig. 1b, lanes 4 and 5). Sequencing 22 elements (AccN\# AJ009223-AJ009233 and AJ009237-AJ009247) confirmed that the 1.3-kbp fragments were full-length transposons. The three 1.2kbp fragments sequenced from the human genome contained a small deletion (AccN\# AJ009234-AJ009236). This suggested that the 0.6-kbp fragments were Hsmarl elements with internal deletions.

The numbers of mammal marl elements were obtained by dot blot/hybridization developed with the mammal marl probe. There were about 1000 mammal marl elements in humans, 20-50 in the mouse, 3000 in the sheep, and 3500 in the cow. Similarly, hybridizations performed with the ITR probe indicated that there were $7000,1000,10,000$, and 10,000 ITRs in the human, mouse, sheep, and cow genomes. As each MLE had two ITRs, there are over 5000 free ITRs in the human genome. This agrees with the presence of 80-bp paired ITR structures and single ITRs (Morgan 1995; Robertson and Zumpato 1997). The excesses of about 900 ITRs in the mouse, 3500 ITRs in the sheep, and 3000 ITRs in the cow suggest that there are also many single ITRs. 
Features of mammal mar1 consensus sequences. The 25 sequences of the full-length and 1.2-kbp mammal marl elements (Acc\# AJ009223-AJ009247) from the four species were aligned (the alignment is available under DS34739 from FTP.EBI.AC.UK in directory pub/databases/embl/align). The consensus sequence in each species was deduced, taking into account the nature of the most frequent base at each position. No full-length ORF encoding a transposase was found, because there were $6-8$ stop codons at the same positions in the four consensus sequences. These stop codons were due to cytosine-thymidine (C-T) transitions occurring in six CpG dinucleotides (positions 227, 470, 663, 670, 715, and 740), one CpT dinucleotide (position 874), and one $\mathrm{CpC}$ dinucleotide (position 989). The four consensus sequences were modified to obtain a putative ORF encoding the transposase, then called Cons Oa, Cons $\mathrm{Mm}$, Cons $\mathrm{Bt}$, and ConsHs.

Within-species phylogenies of mammal mar1 elements. The phylogenetic relationships between mammal marl elements were first analyzed within each species. Two files were set up for each species in order to use the maximum data in the databank. The first contained full-length nucleotide sequences, whereas the second contained nucleotide sequences (about $510 \mathrm{bp}$ long) corresponding to the region encoding the transposase between the two conserved amino acid domains, WVPHEL and YSPDLAP. We used an invertebrate MLE sequence from the entomoparasitic nematode Heterorhabditis bacteriophora as outgroup (Grenier et al. 1999). This MLE has a mean nucleotide similarity of $70.5 \%$ with the complete U52077 sequence and appeared to be the full-length element most closely related to the mammal marl element in Blast searches performed with the databanks.

Twelve full-length Hsmarl elements extracted from the human sequences in the Genbank, (called hsG1 to hsG12), and the Robertson's Hsmarl consensus sequence (Acc\# U52077) were included in the first file with the sequences of our seven full-length elements and the ConsHs sequence. The two consensus trees obtained were similar, the differences being in the number of bootstrap replications supporting nodes. The second file combined the same elements plus five sequences in the databank (Acc\# X85285X84289, Augé-Gouillou et al. 1995). The two consensus trees obtained with this file had many architectural differences and node values lower than those obtained with the first file. This indicates that the nucleotide sequences corresponding to the region encoding the transposase between the WVPHEL and YSPDLAP domains did not contain enough information to reveal the phylogeny of Hsmarl elements. A similar result was obtained with Oamarl sequences, whereas there were no differences between the trees developed from the two files with the Btmarl sequences.

The architecture and the node values in the tree (Fig. 2a) suggested that Hsmarl elements formed at least two groups. All the elements obtained in the present work by PCR amplification except for the hs18 element were in the first group, in which the values at nodes indicated that the relationships were well defined (Fig. 2a, elements in the black square). All the sequences extracted from the databank (hsG1 to hsG12) plus the hs18 elements were in the second group (Fig. 2a, elements in the grey square). The low values at nodes indicated that the relationships between these elements were poorly defined. Their location close to the root of the tree, the branch lengths, and the width of their distribution in the tree indicated that they were more divergent than those of the first group. This indicates that the second group of elements is probably older than the first.

Analysis of the tree developed with the Oamarl elements also suggested two groups (Fig. 2a, elements in the black and grey squares). But one of these group contained only one element, oa1. Two groups might be defined by the phylogenetic analysis of the Btmarl elements (Fig. 2b, elements in the black and grey squares).
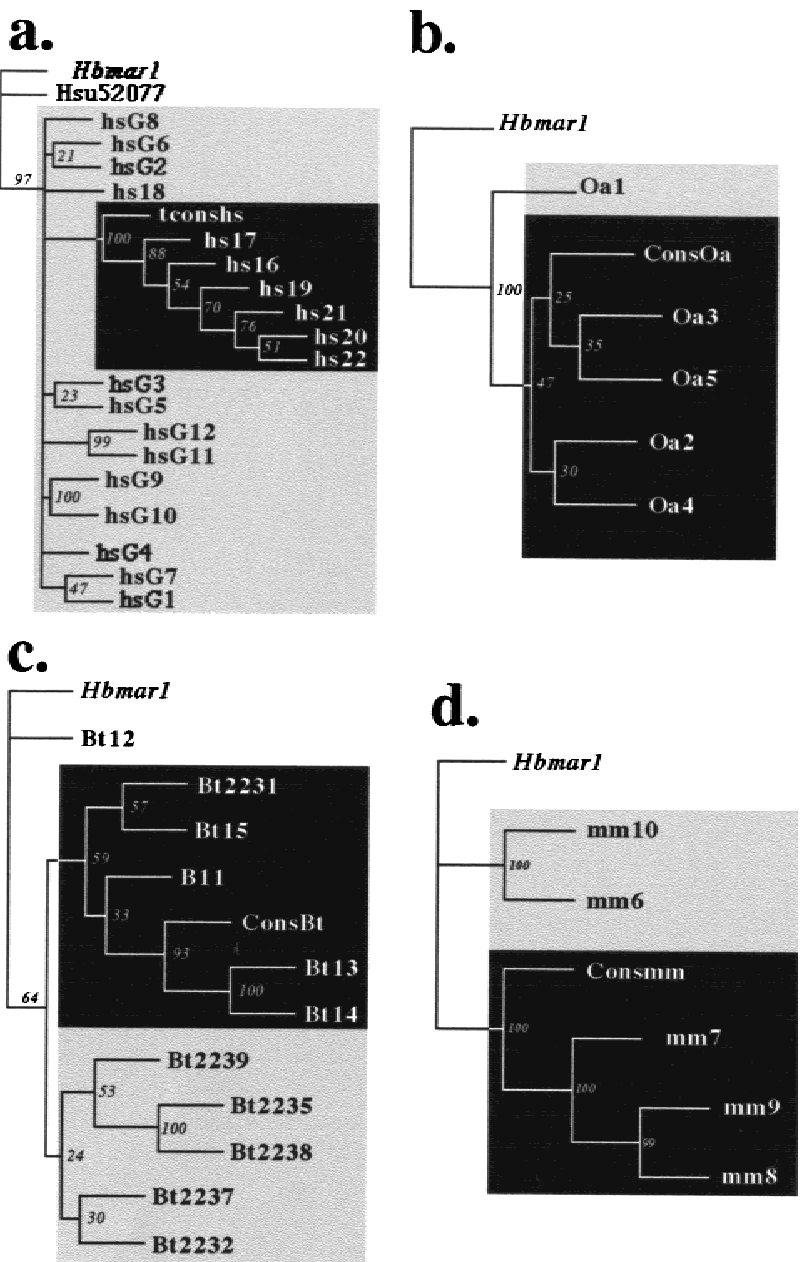

Fig. 2. Consensus trees of the phylogenetic relationships between mammal marl elements from the human (a), cow (b), sheep (c), mouse (d) genomes and resulting from neighbor-joining computations. The number for each hs, $\mathrm{bt}$, oa, and $\mathrm{mm}$ sequence corresponds to those in the alignment available under the accession number DS34739 from FTP.EBI.AC.UK in directory pub/databases/embl/align. hsG1 to hsG12 elements were extracted from 12 sequences (Acc\# Z95124, AC002460, AF029308, Z81009, AJ239318, AC005090, AC006148, AC004063, AC006199, AC004385, AC006143, and Z98746). Hbmarl is the H. bacteriophora MLE outgroup (AccN\# U68392). The trees in $\mathrm{a}, \mathrm{b}$ and $\mathrm{d}$ were developed from the full-length nucleotide sequences of the mammal marl elements. Nucleotide sequences corresponding to the region encoding the transposase between the WVPHEL and YSPDLAP domains were used in $\mathrm{c}$. The groups of related elements in each tree are boxed in black and grey. The values in italics at nodes are the number of times that the branch was present among the 1000 bootstrapped trees. Only nodes having bootstrap values over 20\% were kept.

There may be a third group in this species, but the evidence for it was poor because it consisted of only one element, bt12. Finally, phylogenetic analysis of the murine elements also suggested two groups (Fig. 2c), which were well defined by the topology of the tree and the values at nodes.

Between-species phylogeny of mammal mar1 elements. Analysis of the consensus trees (Fig. 3) confirmed that the Hsmarl and Mmmarl elements were divided into two groups. The locations of the oal and bt12 elements among the hsG1-hsG12 elements also suggested that there were two groups of Oamarl elements, and maybe three groups of Btmarl elements. This consensus tree also revealed that all the elements obtained in the present work by PCR 


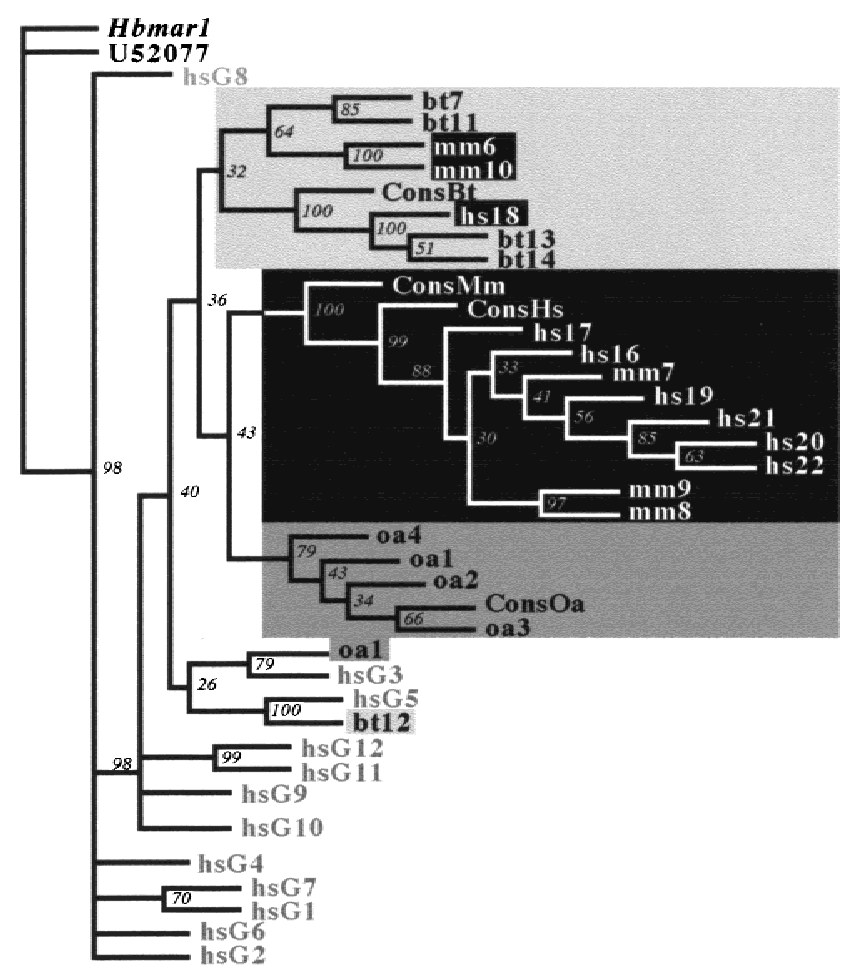

Fig. 3. Consensus tree of the phylogenetic relationships between mammal marl elements from the human (hs), cow (bt), sheep (oa), mouse (mm) genomes. The numbers for each hs, bt, oa, and mm sequence correspond to those in the alignment already used in Fig. 2. Files containing full-length sequences of mammal marl elements were used for Hsmar1, Oamar1, and Mmmarl elements. For Btmarl elements, sequences in the region encoding the transposase between the WVPHEL and YSPDLAP domains were used. The Hsmarl and Mmmarl elements obtained in the present work by PCR amplification are in black boxes and typed in white, whereas the Btmarl are in light grey and Oamarl in dark grey boxes. Hbmarl is the H. bacteriophora MLE outgroup. hsG1 to hsG12 are full-length elements extracted from human sequences found in databanks and typed in grey. The values in italics at nodes indicate the number of times that the branch was present among the 1000 bootstrapped trees. Only nodes with bootstrap values over $20 \%$ were kept.

amplification from the human, sheep, cow, and mouse genomes were in the same part of the tree (Fig. 3, elements in the different squares). The values at nodes distributing these elements suggested that the relationships between at least two populations were well defined in each species.

The Hsmar1 populations in the human genome. Our phylogenetic results suggested that there were at least two populations of mammal marl elements in the four mammalian genomes. However, while the phylogenetic analyses are a good way to represent genetic distances between elements, they are not appropriate for defining populations. Their analysis only allows us to suggest the presence of population. The mammal marl sequences were, therefore, analyzed by mean sequence similarities, GC contents, and dinucleotide frequencies in graph analyses. They confirmed, statistically, the real presence of mammal marl populations in these species. The best criteria for defining populations of mammal marl elements were their $\mathrm{CpG}$ obs/exp ratios and their percentage of sequence similarity with the consensus of their own species.

Three sets of data were checked for Hsmarl populations in the human genome. The first was in the 10 Hsmarl elements obtained in the present work (Acc\# AJ009223-AJ009247), the 12 hsG1hsG12 elements, and the five shorter nucleotide sequences (Acc\# X85285-X84289). Two groups of Hsmarl elements were distin- guishable in the graph (Fig. 4a), showing the relationships between the percentages of nucleic acid similarity of each of these Hsmarl elements with the Cons Hs sequence (vertical axis), and their $\mathrm{CpG}$ exp/obs ratios (horizontal axis). The first contained the hs16, hs17, and hs19-hs22 elements, whereas the 22 others were in the second. These groups had already been observed in our phylogenetic analyses (Fig. 2a). The density ellipses reflecting the features of each of these two groups were drawn with a probability equivalent to $95 \%$ (Fig. 4a, black line) and $99 \%$ (Fig. 4a, grey line) confidence limits. The absence of overlapping regions between the ellipses of the two groups confirmed that there were two distinct Hsmarl populations.

In total, 39 sequences of $600-1200 \mathrm{bp}$ and corresponding to truncated Hsmarl elements were extracted from GenBank and placed in the second data set. A similar graphical analysis was performed (Fig. 4b), which defined only one population of Hsmarl elements. The features of the density ellipses for this population were similar to those of the second Hsmarl population defined in our first analysis (Fig. 4a).

A third set of 46 Hsmarl sequences $460-800$ bp long was extracted from the GenBank. All these human sequences were referenced as transcribed (messenger RNA and ESTs). Analysis of the graph representing their features (Fig. 4c) revealed a single Hsmarl population containing most of the sequences (45/46). Its features indicated that it was the same as the common population in the first (Fig. 4a) and second (Fig. 4b) analyses. Only one sequence (Acc\# AI866805) did not meet the features of this population in this third analysis. Its similarity to the ConsHs sequence (90\%) suggested that it was related to the population containing the hs16, hs17, and hs19-hs22 elements in the first analysis (Fig. 4a, 90-100\%).

In conclusion, our analysis of sequences from different sources (108 in total) showed that there are at least two populations of Hsmarl elements. The amounts of elements in each of these populations cannot be defined. This is owing to the biases linked to our two sources of Hsmarl sequences (euchromatic sequences in Genbank and PCR fragments). The means of nucleotide sequence similarity within each of these populations were $81 \% \pm 6$ for the population common to the three analyses and $95.5 \% \pm 2$ for the population containing the hs16, hs17, and hs19-hs22 elements. This difference showed that the two Hsmarl populations have different ages. Thus, we called the older of these populations Hsmarl-1 and the more recent population Hsmarl-2 (Fig. 4d).

Mammal mar1 populations in the sheep, cow, and mouse genomes. The results for the sheep, cow, and mouse mammal marl elements (Fig. 5a, b, and c) were obtained by using our present data and published sequences (Augé-Gouillou et al. 1995). There may be two populations of mammal marl elements in the sheep genome (Fig. 5a). In agreement with the phylogenetic tree (Fig. 3), one of these populations contained only one sequence (Oa1). The position of this Oamarl element in the general phylogenetic tree (Fig. 3) suggested that it originated from a population older than that containing the other Oamarl elements. These two groups were called Oamar1-1 (Oa1) and Oamar1-2 (Oa2-Oa5 and Oa2202Oa2213).

Phylogenetic analyses of the Btmart1 elements (Figs. 2c, 3) suggested that there were three populations in the cow genome. Our graphic analysis showed only two populations with very similar sequence features (Fig. 5b). The first population contained the Bt2231-Bt2239 elements, while the second contained the Bt11Bt15 elements. Although they are probably very similar in age, we called them Btmarl-1 and Btmarl-2.

Few sequences are available for the mouse genome, but the results clearly showed that there were at least two groups. The group containing mm6 and mm10 elements was called Mmmarl-1 

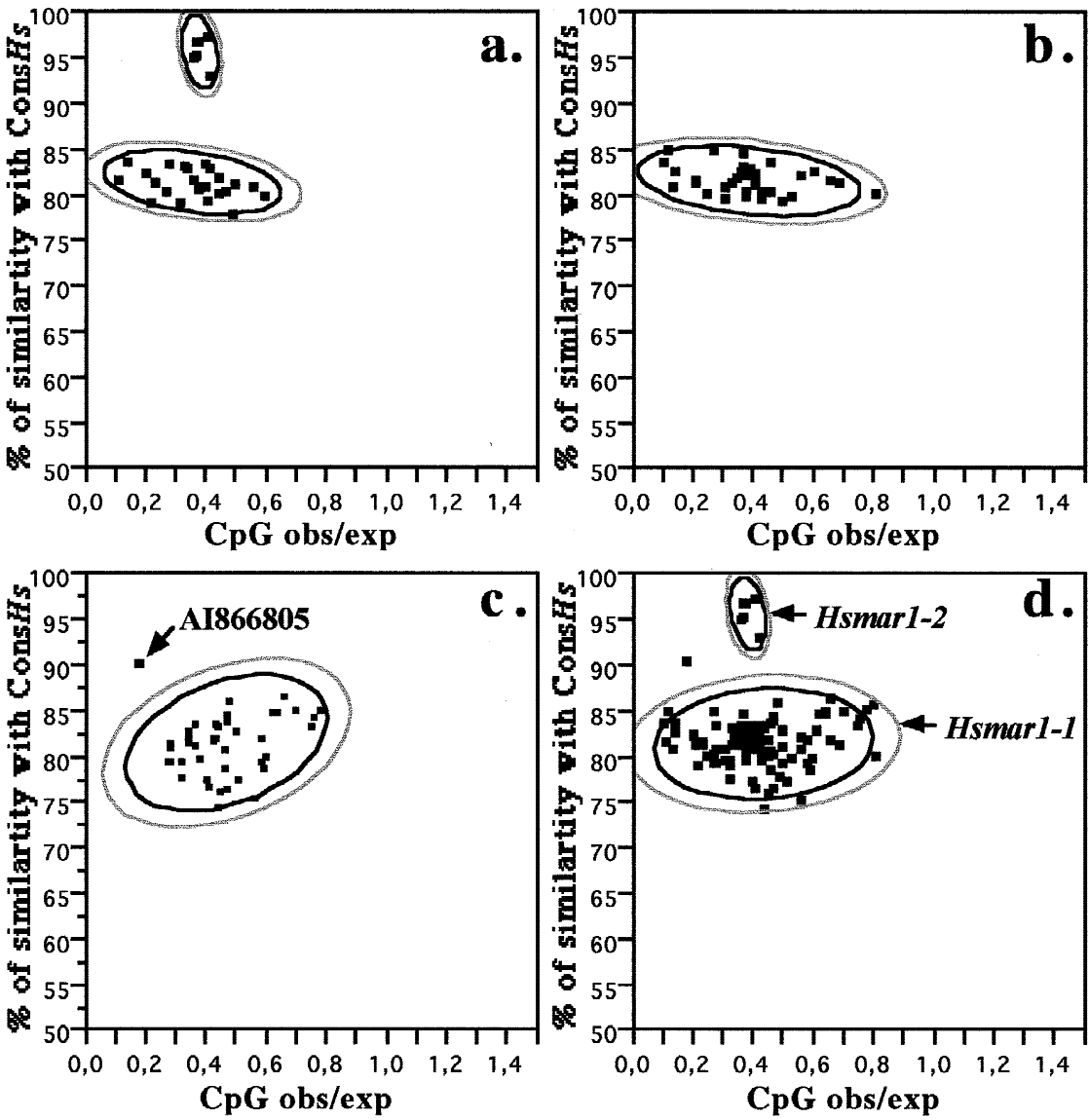

Fig. 4. Graphs of the relationships between the percentage of nucleic acid sequence similarity of each Hsmarl sequence to the consensus sequence ConsHs (vertical axes), and their CpG exp/obs ratio (horizontal axes). (a) uses the 10 Hsmarl elements obtained in the present work (Acc\# AJ009223-AJ009247), Robertson's consensus sequence U52077, the full-length elements hsG1-hsG12, and the five nucleotide sequences corresponding to the region encoding the transposase between the WVPHEL and YSPDLAP domains (Acc\# X85285-X84289). (b) uses all the sequences of truncated Hsmarl elements extracted from Genbank. (c) uses all the human sequences corresponding to transcribed sequences and extracted from Genbank. (d) contains all the data used in (a), (b) and (c). The black and grey ellipses locate the groups of mammal marl elements discriminated in these analyses with a confidence of $95 \%$ and $99 \%$, respectively. The names given to each mammal marl population are indicated. elements, and the one containing $\mathrm{mm} 7, \mathrm{~mm} 8$, and $\mathrm{mm} 9$ elements was called Mmmarl-2 elements.

\section{Discussion}

The number of mammal marl elements in the human, mouse, sheep, and cow genomes varies from about 20-50 to 1000-3500. The number of ITRs related to these elements varied from 900 in the mouse, 7000 in the human, to 10,000 in the sheep and cow genomes. As observed for SINEs (B1 and B2) and L1 LINEs (Duret et al. 1995), they are much less numerous in the mouse genome, and only few full-length elements are detected. Mammal marl elements are also not all the same size in the human, sheep, and cow. There are variable amounts of full-length and internally deleted elements in each species. The internal deletions are not the only source of length variations. Analyses of the databank sequences reveal that many are $5^{\prime}$ or $3^{\prime}$ truncated copies.

The analyses of the elements in each species indicate that there are at least two populations of mammal marl elements in human (Hsmar1-1 and Hsmar1-2), mouse (Mmmarl-1 and Mmmar1-2), sheep (Oamarl-1 and Oamar1-2) and cow (Btmarl-1 and Btmarl2). The location of the elements related to the Hsmarl-1 and Oamarl-1 populations in the phylogenetic tree and their great variations in sequence indicate that these two groups are more ancient than are the Hsmarl-2 and Oamarl-2, respectively. Our results indicate that there are two populations of mammal mar-1 elements of mouse or cow origins. The presence of several populations of mammal mar-1 elements in these mammalian species suggests that they came from several expansions of one or a few elements at different times during the evolution of their respective host ancestors. This indicates that the MLE in mammalian genomes have a life and death cycle similar to those of the AluI SINES (Shen et al. 1991). We find mammal mar-1 elements in rodents, primates, and artiodactyls. This indicates that the mammal marl elements in the genome of the common ancestor of all these species might be older than $50 \mathrm{Myr}$ and arose at least more than 100 million years ago (Springer 1997). We detected no mammal marl elements older than those related to the Hsmar1-1, Oamar11, Btmar 1-1, and Mmmar1-1 populations in our studies. However, if such elements are present in mammalian genomes, their sequences are probably less than 60-70\% similar. Such similarities prevent their detection in Fasta or Blast searches because they are identical to the mean nucleotide sequence similarity between Hsmarl and Hsmar2 elements (52-68\%).

Our explanation for the presence of several mammal marl populations in the mammalian genomes appears to be parsimonious and minimizes horizontal transfers as previously recommended in the literature (Capy et al. 1996; Hartl et al. 1997). However, it does not answer a controversial observation obtained by comparing the invertebrate and mammalian MLEs related to the cecropia sub-family. Our analysis reveals that the older Hsmarl in the common ancestor of the rodents, primates, and artiodactyls is less than 60-70\% identical to the Hsmarl-1 and Hsmarl-2 elements. Moreover, Hsmarl-1 elements are only $75-85 \%$ similar to the Hsmarl-2 elements. Robertson and Zumpano (1997) reported that the invertebrate MLEs in Carpelius sp., Hydra littoralis, and $H$. bacteriophaga genomes are $70-85 \%$ similar to the Hsmarl-2 elements. These observations do not necessarily raise any question about the pattern that we propose here for the development of mammal marl in the mammalian genomes. However, we cannot reasonably explain the processes producing such conservation between so different organisms without invoking horizontal transfers, at least once from vertebrates to invertebrates.

Several features described here for Hsmarl differ from those previously published and resulting from only databank analyses (Robertson and Zumpano 1997). A recent study of the distribution 

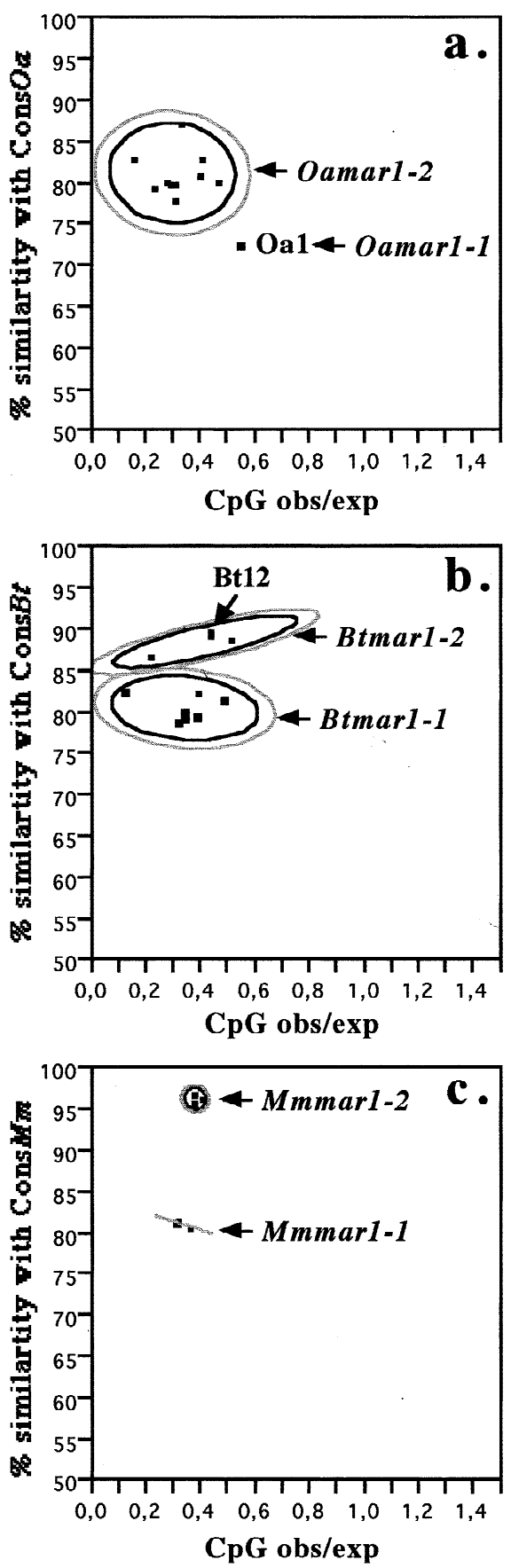

Fig. 5. Graphs of the relationships between the percentage of nucleic acid sequence similarity of each mammal marl sequence to its consensus sequence, ConsOa (a), ConsBt (b), and ConsMm (c) respectively (vertical axes), and their $\mathrm{CpG}$ exp/obs ratio (horizontal axes). The sequences of the region encoding the transposase between the WVPHEL and YSPDLAP domains were used in (a) and (b). They were extracted from the sequences (Acc\# AJ009242-AJ009246 + X84290-X84295) in (a) and (Acc\# AJ009223-AJ009226 + AJ009247 + X84296-X84301) in b. (c) uses the full-length sequences, AJ009237-AJ009241. The black and grey ellipses locate the groups of mammal marl elements identified in these analyses with a confidence of $95 \%$ and $99 \%$, respectively. The names given to each mammal mar1 population are indicated.

and frequency of repeated sequences (Schug et al. 1998) found that the DNA sequences deposited in databanks are biased towards the euchromatic regions expressed. It may thus be inappropriate to estimate the frequencies of repeated sequence families in the total genome of a eukaryotic species by dividing the number of repeated sequences identified by the total amount of sequence surveyed. Thus, if a family of transposable elements is preferentially interspersed in the heterochromatin or in weakly expressed euchromatic regions, the sequence analyses may be greatly biased owing to the kind of sequences in the databanks (our data suggest that this is the case for Hsmarl elements). This may have consequences for detecting transposable elements, establishing a consensus sequence, and estimating their number and diversity and for understanding their evolution.

Finally, we obtained these conclusions only because we compared the results of databank analyses and PCR amplification experiments. Thus, the difference in the number of the Hsmarl elements in the human genome (Robertson and Zumpano: 200; ours: 1000) reflects the fact that these transposons may not be randomly distributed in the human genome, as are other human transposable elements (Chen and Manuelidis 1989).

Acknowledgments. This work was supported by grants from the CNRS (UPRES-A 6035), MENRT, INRA, and from the Genome project. The English text was edited by Dr. C.O. Parkes.

\section{References}

Augé-Gouillou C, Bigot Y, Pollet N, Hamelin MH, Meunier-Rotival M et al. (1995) Human and other mammalian genomes contain transposons of the mariner family. FEBS Lett 368, 541-546

Capy P, Bazin C, Anxolabéhère D, Langin T (1996) Horizontal transfer and the evolution of tranposable elements. In: Transgenic Organisms and Biosafety, Schmidt ER, Hankeln T (eds). (Berlin, Heidelberg: Springer-Verlag), pp. 15-30

Chen TL, Manuelidis L (1989) SINEs and LINEs cluster indistinct DNA fragments of Giemsa band size. Chromosoma 98, 309-316

Dessen C, Fondrat C, Valencien, Mugnier C (1990) A French service for access to biomolecular sequence databases. Cabios 6, 355-356

Duret L, Mouchirous D, Gautier C (1995) Statistical analysis of vertebrate sequences reveals that long genes are scarce in GC-rich isochores. J Mol Evol 40, 308-317

Grenier E, Abadon M, Brunet F, Capy P, Abad P (1999) A Mariner-like transposable element in the insect parasite nematode Heterorhabditis bacteriophaga. J Mol Evol 48, 328-336

Hartl DL, Lovoskaya ER, Nurminsky DI, Lohe AR (1997) What restricts the activity of mariner-like transposable elements? Trends Genet 13 , 197-201

Mahillon J, Chandler M (1998) Insertion sequences. Microbiol Mol Biol Rev 62, 725-774

Maruyama K, Schoor KD, Hartl DL (1991) Identification of nucleotide substitutions necessary for trans-activation of mariner transposable elements in Drosophila: analysis of naturally occurring elements. Genetics $128,777-784$

Morgan GT (1995) Identification in the human genome of mobile elements spread by DNA-mediated transposition. J Mol Biol 254, 1-5

Oosumi T, Belknap WR, Garlick B. (1995) Mariner transposons in humans. Nature 378, 672

Plasterk RHA, Izsvak Z, Ivics Z (1999) Resident aliens. Trends Genet 15 , 326-332

Rabouam C, Comes AM, Bretagnolle V, Humbert JF, Periquet G et al. (1999) Features of DNA fragments obtained by random amplified polymorphic DNA (RAPD) assays. Mol Ecol 8, 493-503

Robertson HM, Martos R (1997) Molecular evolution of the second ancient human mariner transposon, Hsmar2, illustrates patterns of neutral evolution in the human genome lineage. Gene 205, 219-228

Robertson HM, Zumpano KL (1997) Molecular evolution of an ancient transposon, Hsmarl, in the human genome. Gene 205, 203-217

SAS Institute (1995) JMP Statistics software3.1 SAS Institute

Schug MD, Wetterstrand KA, Gaudette MS, Lim RH, Hutter CM, Aquadro CF (1998) The distribution and frequency of microsatellite loci in Drosophila melanogaster. Mol. Ecol. 7, 57-70

Shen RM, Batzer MA, Deininger PL (1991) Evolution of the master Alu gene(s). J Mol Evol 33, 311-320

Springer MS (1997) Molecular clocks and timing of the placental and marsupial radiations in relation to the cretaceous-tertiary boundary. $\mathrm{J}$ Mamm Evol 4, 285-295 